This is the Accepted Version of a paper published in Internal Medicine Journal:

Tan, Joanne Yue-Ai, Otty, Zulfiquer A., Vangaveti, Venkat N., Buttner, Petra, Varma, Suresh C., Joshi, Abhishek J., Kelly, Jenny, Collins, Michael, and Sabesan, Sabe S. (2016) A prospective comparison of times to presentation and treatment of regional and remote head and neck patients in North Queensland, Australia. Internal Medicine Journal, 46 (8). pp. 917-924.

http://dx.doi.org/10.1111/imj.13138 


\section{A prospective comparison of times to presentation and treatment of regional and remote head and neck cancer patients in North Queensland, Australia}

Joanne Yue-Ai Tan 1,2,

Zulfiquer. A. Otty ${ }^{2,3}$,

Venkat N.Vangaveti ${ }^{3}$,

Petra Buttner ${ }^{3,4}$,

Suresh C. Varma ${ }^{2,3}$,

Abhishek J. Joshi ${ }^{2,3}$, Jenny Kelly ${ }^{3}$,

Michael Collins ${ }^{2,3}$

Sabe S. Sabesan ${ }^{2,3}$
Medical Oncology Advanced Trainee Registrar

Staff Specialist Medical Oncologist

Research Fellow/Biostatician

Biostatician

Staff Specialist Medical Oncologist

Staff Specialist Medical Oncologist

Senior Research Fellow

Staff Specialist Radiation Oncologist

Director of Medical Oncology and Clinical Dean of Northern training network clinical school

1. Cancer Care Services, Royal Brisbane and Women’s Hospital, Butterfield Street, Herston QLD 4029, Australia.

2. Cancer Care Services, The Townsville Hospital, 100 Angus Smith Drive, Douglas, QLD 4814, Australia.

3. College of Medicine and Dentistry, James Cook University, Townsville, QLD 4811, Australia.

4. Tropical Health Solutions Pty Ltd, 72 Kokoda St, Idalia, Townsville QLD 4811, Australia

5. Centre for Nursing and Midwifery Research, James Cook University, Townsville, QLD 4811, Australia

\section{Correspondence to:}

J.Y. Tan

Department of Medical Oncology, Cancer Care Services, The Royal Brisbane and Women's Hospital, Butterfield St, Herston QLD 4029, Australia.

Telephone No +61 736468111

Email: joanne.tan2@health.qld.gov.au

(formerly of Cancer Care Services, The Townsville Hospital, 100 Angus Smith Drive, Douglas QLD 4814, Australia.

And James Cook University, Townsville, QLD 4811, Australia.)

\section{Acknowledgements}

We wish to thank our research nurse Jill Matthiesen for her contribution to the study. This project received funding from the Townsville Health Service District Private Practice Study, Research and Education Trust Fund. (Not for Profit Benevolent Trust Fund).

A provisional abstract of this study was presented at ASCO Annual Meeting 2014 (30 ${ }^{\text {th }}$ May to $3^{\text {rd }}$ June) by Z. Otty. J Clin Oncol 32:5s, 2014 (suppl; abstr 6539)

http://meetinglibrary.asco.org/content/126036-144

There are no relevant conflicts of interest declared for any of the authors

This article has been accepted for publication and undergone full peer review but has not been through the copyediting, typesetting, pagination and proofreading process, which may lead to differences between this version and the Version of Record. Please cite this article as doi: 10.1111/imj.13138

This article is protected by copyright. All rights reserved. 
Introduction: This study aims to examine differences between outer regional (OR) and remote/very remote (RVR) patients in northern Queensland, Australia, in the times taken to receive various aspects of head and neck cancer management.

Methods: Our study prospectively recruited head and neck cancer patients presenting to three North Queensland regional hospitals from 1/2009 to 1/2011. Data on demographic and cancer specific details, co-morbidities and timing of presentation to various services were collected using a self-administered questionnaire that included two questions in relation to possible reason for delays to health services. Multivariate linear regression analyses were conducted to assess the effects of various demographic characteristics on time delays. Survival and disease recurrence data were analysed in 2014.

Results: 158 patients participated. RVR patients had significantly longer median times between diagnosis and first treatment compared with OR patients $(\mathrm{p}=0.015)$. Indigenous patients had significant delays from diagnosis to first treatment $(\mathrm{p}=0.013)$ and visit to first specialist and treatment $(\mathrm{p}=0.031)$ compared to non-Indigenous patients. Longer median times between symptoms and first treatment associated with low income $(p=0.03)$ and lower education level $(\mathrm{p}=0.04)$. Disease recurrence was higher for RVR patients compared with OR patients ( $\mathrm{p}=0.04)$, without significant differences in overall survival. Possible reasons for delays included patient and professional factors.

Conclusions: Significant delays in various aspects of head and neck cancer management were associated with remoteness, Indigenous and socioeconomic status. While patient and professional factors could be addressed at local levels, it requires a state and national level approach for sustainable improvement in outcomes.

Key words: Head and neck cancer, rural health, diagnosis delay, treatment delay

This article is protected by copyright. All rights reserved. 


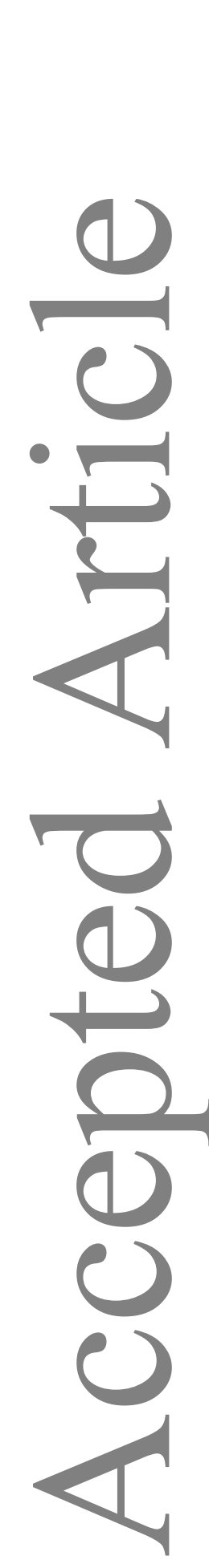

\author{
Abbreviations: \\ ASGC = Australian Standard Geographical Classification \\ $\mathrm{CI}=$ Confidence Interval \\ ECOG = Eastern Cooperative Oncology Group \\ ENT = Ear, Nose and Throat (Otolaryngology) Surgeon \\ $\mathrm{OR}=$ Outer Regional \\ RVR = Remote $/$ Very Remote \\ TNM staging $=$ Tumour, Nodes, Metastases staging
}

Introduction: This study aims to examine differences between outer regional (OR) and remote/very remote (RVR) patients in northern Queensland, Australia, in the times taken to receive various aspects of head and neck cancer management.

Methods: Our study prospectively recruited head and neck cancer patients presenting to three North Queensland regional hospitals from 1/2009 to 1/2011. Data on demographic and cancer specific details, co-morbidities and timing of presentation to various services were collected using a self-administered questionnaire that included two questions in relation to possible reason for delays to health services. Multivariate linear regression analyses were conducted to assess the effects of various demographic characteristics on time delays. Survival and disease recurrence data were analysed in 2014.

Results: 158 patients participated. RVR patients had significantly longer median times between diagnosis and first treatment compared with OR patients $(\mathrm{p}=0.015)$. Indigenous patients had significant delays from diagnosis to first treatment $(\mathrm{p}=0.013)$ and visit to first specialist and treatment $(\mathrm{p}=0.031)$ compared to non-Indigenous patients. Longer median times between symptoms and first treatment associated with low income $(p=0.03)$ and lower education level $(\mathrm{p}=0.04)$. Disease recurrence was higher for RVR patients compared with 
OR patients $(\mathrm{p}=0.04)$, without significant differences in overall survival. Possible reasons for delays included patient and professional factors.

Conclusions: Significant delays in various aspects of head and neck cancer management were associated with remoteness, Indigenous and socioeconomic status. While patient and professional factors could be addressed at local levels, it requires a state and national level approach for sustainable improvement in outcomes.

\section{Introduction:}

Head and neck cancer describes a range of cancers to the oral cavity, salivary glands, nasal cavity, sinuses, pharynx and larynx ${ }^{1-4}$. The most common histological type is squamous cell carcinoma $^{1-4}$. Despite recent advances in treatment, mortality and morbidity rates continue to be high, particularly for rural and remote patients ${ }^{3,5}$. These high rates may be due to a number of factors, including delays in diagnosis and treatment ${ }^{6}$. Between 2006-2010, the overall five -year survival rate for head and neck cancer patients in Australia was 68.2\%. ${ }^{3}$

People living in regional and remote areas of Australia have poorer health outcomes including lower survival rates when compared to their city counterparts ${ }^{7-10}$. Some of the suggested reasons for this disparity include increased cancer risk factors, including smoking, alcohol consumption and sun exposure amongst people in regional and remote areas ${ }^{7-10}$. Other reasons may include socio-economic factors and poorer access to health care services ${ }^{8}$. The rural/remote disadvantage is particularly evident in Australia with its large geographical 
area and relatively small population. Health services are often stretched to the limit in rural and remote areas. North Queensland encompasses a very large geographical area $\sim 740000 \mathrm{~km} 2$ ( $\sim 43 \%$ of Queensland) but serves a population of only $\sim 600000$, ( $15 \%$ of the total population of Queensland) ${ }^{11}$. This large area is serviced by three tertiary hospitals in Townsville, Cairns, and Mackay, with the main specialist centre in Townsville.

The usual management pathway for head and neck cancer begins with the patient presenting to their general practitioner (primary health care physician) following symptoms ${ }^{1,2,12,13}$. Generally after clinical review, imaging scans are undertaken prior to referral to specialists (e.g. surgeons and/or interventional radiologists) for biopsy and further management. Once a diagnosis is confirmed, the patients are referred to head and neck multidisciplinary meetings and clinics (ENT surgeons, radiation oncologists, medical oncologists) where optimal treatment is planned. Treatments may include surgical excision, radiation therapy, chemotherapy and/or a combination of these ${ }^{1,2}$. Treatment may be either with curative or palliative (for symptom relief) intent.

Delays in treatment can be classified according to 'patient' or 'professional' delays ${ }^{6,13}$. Patient delays are where patient factors cause delays from the onset of their first symptoms until presentation to a health care provider for treatment (e.g. patients dismissing their symptoms until at an advanced stage $)^{6,13}$. Professional delays are those where there are health care provider delays from consultation to diagnosis to treatment ${ }^{6,13}$ (e.g. delay until treatment such as surgery or radiation able to be booked and performed). The average patient delays ranged from 1.0 to 5.4 months and the average professional delays from 3 to 21 weeks 6, 13. The majority of published studies were retrospective case control studies that aimed to

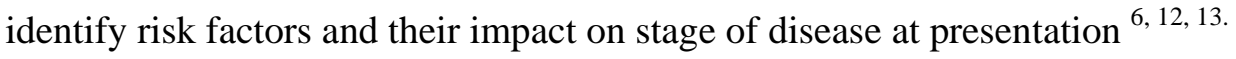

This article is protected by copyright. All rights reserved. 
The identification of specific barriers to health services will inform the development of strategies to improve health outcomes for patients from rural and remote areas. There is a paucity of literature that explores the specific delays patients from rural and remote areas with head and neck cancer experience in the Australian setting. This study aims to examine the differences between patients from outer regional areas and those from remote areas in the time taken to receive head and neck cancer management by the North Queensland Cancer Care Services.

This article is protected by copyright. All rights reserved. 


\section{Methods:}

\section{Participants and Recruitment}

Newly diagnosed head and neck cancer patients presenting to the Townsville, Cairns and Mackay Hospitals were prospectively recruited over a 24-month period from 1/2009 to 1/2011 following identification through specialist outpatient clinics and multidisciplinary head and neck team meetings and informed consent. Ethics approval was granted by the participating health services.

\section{Data Collection}

Data was collected through self-administered questionnaires as well as review of medical records, pathology reports and the existing MOSAIQ ${ }^{\mathrm{TM}}$ clinical oncology database which is used for integrated cancer services provision. The questionnaire included the following details:

(1) Demographic and socio-economic details such as age, gender, ethnicity, Indigenous status, gross household income, highest level of education, living arrangements, employment, private health insurance and place of residence.

(2) Distance from home to the General Practitioner, local hospital, nearest specialist for surgery, chemotherapy, radiation therapy and palliative care.

(3) Co-morbidities, medications, pre-diagnosis symptoms and performance status.

(4) Dates of first presentation to a general practitioner, investigations, specialist reviews and treatment. Remoteness was classified according to Australian Standard Geographical Classification (ASGC) remoteness classification into outer regional and remote/very remote categories $^{14}$.

This article is protected by copyright. All rights reserved. 
At the end of the questionnaire, patients were asked to respond to two short answer questions “why was there a delay in reporting your first symptoms to a doctor?” and Why do you think you felt it too difficult to get treatment?’. Survival and disease recurrence data was examined at April 2014. Patients were classified as lost to follow up if there was no patient information recorded since September 2013.

\section{Data Analysis}

We used the Statistical Package for Social Sciences (SPSS v22) for analysis. Fisher's test was used to identify differences between the outer regional and remote/very remote cohorts. Kruskal-Wallis and Mann-Whitney tests were used to calculate the p-values when examining differences in time delays experienced between stage, Indigenous status, and socio-economic status. Chi squared tests were used for survival and disease recurrence data. Multivariable linear regression analyses were conducted to assess the effects of demographic characteristics (age, gender, Indigenous status, level of education, employment status, living arrangement, number of dependents, private health insurance, synchronous and previous cancer, and income) on time delays. Times were logarithmically transformed to achieve approximate normal distributions. Statistically significant influencing factors were identified using backward and forward modelling processes. Remaining characteristics were checked for potential confounding factors (changes in estimator by at least 5\%). Models were adjusted for identified confounders. Data was also analysed according to whether the patient was from Townsville (the main tertiary hospital), Mackay or Cairns (both smaller, hospitals), compared with other remote areas. The stage of head and neck cancer, gender, age ( $<50,51$ to $70,>70$ ) Indigenous status and socioeconomic status (gross household income, highest level of education) were also examined.

This article is protected by copyright. All rights reserved. 


\section{Results:}

One hundred and fifty-eight (158) head and neck cancer patients were identified from January 2009 to January 2011 (Table 1). All of these patients had squamous cell carcinomas. The majority of patients had advanced disease, with 33 patients (21\%) stage III, and 83 patients (52\%) stage IV at the time of diagnosis. In our study group, stage IV head and neck cancers included patients who had locoregionally advanced disease without evidence of distant metastasis and were treated with curative intent. One hundred and eighteen (118) patients (75.3\%) received curative intent treatment and 40 patients (25\%) received palliative treatment (Table 1). Sites of primary cancer and corresponding patient numbers are as follow: oral cavity 83 (53\%), pharynx 30 (19.0\%), larynx 26 (15.6\%), salivary glands $12(6.8 \%)$ and nasal cavity and paranasal sinus 7 (4.3\%). Top five predominant symptoms (with corresponding patient numbers) were pain (99), obvious lump (85), dysphagia (81), voice change (57) and sore throat (54). No differences in the distribution of sites of primary cancer or symptoms were observed between rural and urban groups.

Upon further analysis in 2014, with follow up between $3-5$ years, overall 83 patients (52.5\%) were alive, 10 patients (6.3\%) were lost to follow up and 65 patients $(41.1 \%)$ had died. Of the curative treatment group, 31 patients (26.3\%) had died. The median survival time of these 31 deceased patients was 20.5 months (overall median survival not yet reached with $>50 \%$ alive). Twenty (20) curative intent patients (16.9\%) had disease recurrence. The median time to disease recurrence was 9 months (range 4- 43 months).

Ninety-eight patients (62\%) were living in outer regional areas and 59 patients (37\%) in remote /very remote areas. One patient identified from a metropolitan area. More Caucasians were living in outer regional areas (89.9\%), compared to those in remote/very

This article is protected by copyright. All rights reserved. 
remote areas (76.3\%). There was no significant difference between TNM stage and whether patients were from outer regional or remote/very remote areas. Patients living in remote/very remote areas compared to outer regional areas experienced significantly longer median times between diagnosis and first treatment $(\mathrm{p}=0.015)$. There was a significant difference in disease recurrence for patients living in remote/very remote areas with 11/31 (35.5\%) compared with patients from outer regional areas 8/56 (14.3\%) [Odds ratio 3.30] (CI 1.15 to 9.42, $\mathrm{p}=0.04)$. However, there was no significant difference between mortality for those living in outer regional or remote/very remote locations.

Forty-two (42) patients were identified as living in the Townsville area, forty-one (41) in the Cairns or Mackay area, and 75 in other areas. There was a significant difference in median times between diagnosis and first treatment (36.5 days, vs 40 days vs 55.5 days, $\mathrm{p}=0.012$ ), and between first specialist visit and first treatment(35.5 days ,vs 42.5 days vs 55 days, $\mathrm{p}=0.003$ ). There were no significant differences in mortality or disease recurrence between these groups. (See appendix 1 online for table regarding Townsville vs Cairns/Mackay vs Other Areas for more detailed data)

When compared with non-Indigenous patients, Indigenous patients had significant delays from diagnosis to first treatment $(\mathrm{p}=0.013)$ and from their first specialist visit to treatment $(\mathrm{p}$ $=0.031)$ (See Table 2). Sixteen out of twenty-three Indigenous patients (69.6\%) presented with Stage IV disease. More Indigenous patients (10/23, 43.5\%) had palliative intent treatment compared with non-Indigenous patients (30/135, 22.2\%). There were no significant differences in mortality rates between Indigenous and non-Indigenous patients.

There was a statistically significant delay in time between onset of symptoms and first treatment in patients with lower income compared to those with higher incomes (95\% CI, 0.785 to $0.036, \mathrm{p}=0.032$ ). Similar results were found in patients with primary or lower level 
of education compared to those with higher education levels (95\% CI 0.011 to $0.817, \mathrm{p}=$ 0.044). Patients without private health insurance also experienced significant delay in the time between diagnosis and first treatment (95\% CI, 1.206 to $0.088, \mathrm{p}=0.024)$. No significant differences in mortality or disease recurrence between patients of different socioeconomic status were found (Table 3).

Analysis based on TNM stage did not find any significant differences between stage and median times between symptoms, first consultation, visit to first specialist, diagnosis and first treatment. Analysis revealed that treatment delays ( $>30$ days) compared to those who had earlier treatment, did not result in significant increased mortality or disease recurrence.

\section{Reason for delays:}

71 patients responded to the two questions in relation the reason for delays. Reasons and corresponding patient numbers are outlined as follow:

Presentation to primary care:

Patients had known that there was something serious but presented late due to the hope that it would go away (34), flooding and cyclone (5) and patients attributing their symptoms to other causes (1)

Delay in specialist services:

Delayed referral by general practitioner due to loss of referral (6), patients failing to attend appointments (9), patients undecided about treatment (9), investigation of other comorbidities (7) and delay in booking surgery (3). No formal comparison between rural and urban patients was conducted due small patient numbers in each subgroup.

This article is protected by copyright. All rights reserved. 


\section{Discussion:}

We believe this is the first Australian prospective study to examine the differences between regional, and remote/very remote head and neck cancer patients' diagnostic, referral and management pathways. Our study found significantly longer times to first treatment for remote/very remote patients when compared to those in regional areas. Patients from remote locations had a trend to longer median times across all aspects of management of their head and neck cancer, although not all aspects reached statistical significance. The delays in presentation was not reflected by significant differences in stage of cancer at diagnosis. Disease recurrence was significantly greater in the remote/very remote group compared to the outer regional group; however, this finding did not translate to statistically significant differences in survival rates. A larger cohort of patients and longer follow up times may be needed to demonstrate any survival differences.

Although the number of Indigenous patients in our study was small, results indicated that these patients were significantly more likely to receive only palliative treatment. More Indigenous, than non-Indigenous patients, presented with Stage IV disease. Delays to presentation to health services are likely to lead to later stage disease at diagnosis. There appeared to be a trend towards longer median time between symptoms and first consultation, however, this finding did not reach statistical significance. The time between diagnosis and first treatment, and first specialist visit and treatment were statistically significantly longer in this patient cohort. Having palliative treatment only may add to the delay in time to first treatment due to the lack of urgency for palliative treatment.

This article is protected by copyright. All rights reserved. 
Indigenous patients have been shown to have poorer health outcomes compared to nonindigenous patients ${ }^{15,16}$. Indigenous liaison officers help provide support and education to patients but ongoing work is needed to help bridge the gap.

Overall, the patient delays reported in our study at 62 days (with a wide range from 0 to 647 days), were similar to those reported in other studies $\left(1.0-5.4\right.$ months (ie. $30-160$ days) ${ }^{6}$, 13. At least $22 \%$ of our patients either assumed that the symptoms would go away or attributed their symptoms to other causes. Our estimated total professional delay from first consultation to first treatment was 94 days (range 2 - 794 days). This delay is consistent with other reports of professional delays of 3 to 21 weeks (i.e. 21 to 147 days) ${ }^{6,13}$. Once diagnosis was confirmed, the median time to first treatment was 42 days (range 0 to 429 days). In our study, nearly $10 \%$ of our patients experienced delays due to loss of referral or failure to attend clinics due to misunderstanding. Longer professional delay may be influenced by patient preferences as well as to when their treatment begins. This issue may be of particular relevance in the case of palliative treatment. Our total median time delay between symptoms and first treatment in our study was 216.5 days.

Delays in treatment are often thought to confer worse outcomes, however, there are some conflicting reports. A Dutch study ${ }^{17}$ reported that treatment delay up to 90 days in head and neck squamous cell carcinomas was not associated with worse prognosis in terms of survival or disease recurrence. A recent meta-analysis found that diagnostic delay increased the mortality of head and neck cancer patients by a factor of 1.34 and referral delay increased mortality by three times, although the overall mortality was not significantly increased ${ }^{6}$.

As the majority of the patients in our study had advanced disease (stage IV - 52.9\%), and we included a variety of tumour sites, our survival rate of $53.5 \%$ at 3 years, and disease recurrence rate of $16.9 \%$, appears to be roughly equivalent with other centres. Most studies 
however report 5 year survival (not 3 year) and report on specific tumour sites. The Oxford Cancer Intelligence Unit reported the three year survival rate for head and neck cancers in England varied from 33 to $75 \%$ depending on tumour site ${ }^{18}$.

One of the issues regional and remote cancer patients experience is timely access to specialist treatment. A recent study looked at Head and Neck outcomes in centres with high volumes of patients accrued in clinical trials vs centres with low volumes of patients, and found that overall survival was worse for the centres with low volumes of patients even after adjustment for prognostic factors and radiotherapy compliance ${ }^{19}$. While it is desirable to have treatment at high volume specialist centres, this is not always practical and may further contribute to treatment delays. Townsville is the major head and neck cancer centre for the North Queensland district. The next major referral centre is located in Brisbane approximately $1350 \mathrm{~km}$ away.

Despite awareness of the rural/remote disparity, there has been little progress in overcoming the rural/remote disadvantage in terms of cancer death rates ${ }^{7-10}$. Various Australian studies, have identified factors contributing to the excessive cancer deaths in regional and remote areas such as higher proportions of Indigenous people, greater economic disadvantage associated with some regional and remote locations, a higher prevalence of cancer risk factors, less cancer screening, delays in seeking medical attention and/or delays in diagnosis, higher prevalence of comorbid conditions and treatment disparities ${ }^{8}$. Some possible strategies to help bridge the rural/regional divide include greater support for regional and remote patients to travel, better managed referral pathways, various specialist outreach models of care such as "fly in fly out" specialists, timely processing of referrals, virtual multidisciplinary teams coordinated by care coordinators, building the capacity of local staff in regional cancer services and telemedicine ${ }^{8}$. 
Telemedicine is the delivery of medical consultations using videoconferencing and other information and communication technologies. Telemedicine has the advantage of bringing the specialist consultations to the patients in their local communities, allowing the patient to avoid travelling long distances to the centre and allowing them to remain at home with their support network ${ }^{19}$. This may assist with expediting timely diagnosis and treatment and follow up.

Addressing rural healthcare workforce shortages is important in order to bridge the regional/remote divide. Increasing the number of adequately trained medical, nursing and other health care professionals in Australia may translate to more health services for regional, rural and remote communities. Education of primary healthcare workers on head and neck cancers may lead to more timely consultations, diagnosis and treatment

Another area for further study is to examine whether there are differences in the percentage of P16 positivity (Human Papilloma Virus (HPV) associated) head and neck cancers between rural/remote cohorts compared to their metropolitan counterparts. P16 positive head and neck tumours have been found to have better prognostic outcomes ${ }^{20}$. Whether this risk factor/pathogenesis differences between rural/remote cohorts compared to their metropolitan counterparts, accounts for some of the disparities is yet to be explored.

In conclusion, our study demonstrated delays in various aspects of head and neck cancer management associated with geographical remoteness and other socio-economic factors and attempted to identify possible reasons for these delays. While some patient and professional factors could be addressed at local levels, a state-wide or national level approach is needed to achieve sustainable outcomes.

This article is protected by copyright. All rights reserved. 
References

1 National Comprehensive Cancer Network (NCCN). NCCN Clinical Practice Guidelines in Oncology: Head and Neck Cancers. V 2.2014 2014. available at http://www.nccn.org/professionals/physician_gls/pdf/head-and-neck.pdf

2 Gregoire V, Lefebvre JL, Licitra L, Felip E, Group E-E-EGW. Squamous cell carcinoma of the head and neck: EHNS-ESMO-ESTRO Clinical Practice Guidelines for diagnosis, treatment and follow-up. Annals of oncology : official journal of the European Society for Medical Oncology / ESMO. 2010; 21 Suppl 5: v184-6.

3 Australian Institute of Health and Welfare (AIHW). Head and neck cancers in Australia. 2014; Cancer series 83. Cat. no. CAN80. Available at http://www.aihw.gov.au/publication-detail/?id=60129547291

4 Cooper JS, Porter K, Mallin K, Hoffman HT, Weber RS, Ang KK, et al. National Cancer Database report on cancer of the head and neck: 10-year update. Head \& neck. 2009; 31: 748-58.

5 Singh J, Jayaraj R, Baxi S, Ramamoorthi R, Thomas M. Incidence and mortality from mucosal head and neck cancers amongst Australian states and territories: what it means for the northern territory. Asian Pacific journal of cancer prevention : APJCP. 2013; 14: 5621-4.

6 Seoane J, Takkouche B, Varela-Centelles P, Tomas I, Seoane-Romero JM. Impact of delay in diagnosis on survival to head and neck carcinomas: a systematic review with metaanalysis. Clinical otolaryngology : official journal of ENT-UK ; official journal of

Netherlands Society for Oto-Rhino-Laryngology \& Cervico-Facial Surgery. 2012; 37: 99106.

$7 \quad$ Phillips A. Health status differentials across rural and remote Australia. The Australian journal of rural health. 2009; 17: 2-9.

8 Coory MD, Ho T, Jordan SJ. Australia is continuing to make progress against cancer, but the regional and remote disadvantage remains. The Medical journal of Australia. 2013; 199: 605-8.

9 AIHW. Rural, regional and remote health: indicators of health status and determinants of health. Cat. no. PHE 97. 2008. Available at http://www.aihw.gov.au/publication-detail/?id=6442468076

10 AIHW. Rural, regional and remote health: a study on mortality. Rural health series no. 8. Cat. no. PHE 95. 2nd edition. Canberra 2007. Available at http://www.aihw.gov.au/publication-detail/?id=6442468076

11 Queensland Health. Health Indicators 2009 - North Queensland. Tropical Population Health Service. Cairns 2009. Available at http://www.health.qld.gov.au/ph/documents/tphn/healthindicatornthqld.pdf

12 Alho OP, Teppo H, Mantyselka P, Kantola S. Head and neck cancer in primary care: presenting symptoms and the effect of delayed diagnosis of cancer cases. CMAJ : Canadian Medical Association journal = journal de l'Association medicale canadienne. 2006; 174: 779-84.

13 Stefanuto P, Doucet JC, Robertson C. Delays in treatment of oral cancer: a review of the current literature. Oral surgery, oral medicine, oral pathology and oral radiology. 2014; 117: 424-9.

14 Australian Bureau of Statistics (ABS). Australian Statistical Geography Standard (ASGS). Volume 5 - Remoteness Structure. Canberra: ABS July, 2011. Available at http://www.ausstats.abs.gov.au/ausstats/subscriber.nsf/0/A277D01B6AF25F64CA257B0300 0D7EED/\$File/1270055005_july\%202011.pdf

This article is protected by copyright. All rights reserved. 
15 Moore SP, O'Rourke PK, Mallitt KA, Garvey G, Green AC, Coory MD, et al. Cancer incidence and mortality in Indigenous Australians in Queensland, 1997-2006. The Medical journal of Australia. 2010; 193: 590-3.

16 Cunningham J, Rumbold AR, Zhang X, Condon JR. Incidence, aetiology, and outcomes of cancer in Indigenous peoples in Australia. The Lancet Oncology. 2008; 9: 58595.

17 van Harten MC, de Ridder M, Hamming-Vrieze O, Smeele LE, Balm AJ, van den Brekel MW. The association of treatment delay and prognosis in head and neck squamous cell carcinoma (HNSCC) patients in a Dutch comprehensive cancer center. Oral oncology. 2014; 50: 282-90.

18 Roche MF, Watters AM, Wight R. Relative survival rates for head and neck cancers in England. Oxford Cancer Intelligence Unit The National Cancer Intelligence Unit. 2010. http://www.ncin.org.uk/view?rid=751

19 Sabesan S, Kelly J, Evans R, S L. A teleoncology model replacing face-to-face specialist cancer care: Perspectives of patients in North Queensland, Australia. Journal of Telemedicine and Telecare 2014; 20: 207 - 2011.

20 Ang KK, Harris J, Wheeler R, Weber R, Rosenthal DI, Nguyen-Tan PF, et al. Human papillomavirus and survival of patients with oropharyngeal cancer. The New England journal of medicine. 2010; 363: 24-35.

This article is protected by copyright. All rights reserved. 


\begin{tabular}{|c|c|c|c|c|}
\hline \multirow[b]{2}{*}{ Characteristic } & \multicolumn{4}{|c|}{ ASGC classification } \\
\hline & $\begin{array}{l}\text { Total } \\
\mathrm{N}=158\end{array}$ & $\begin{array}{l}\text { Outer regional* } \\
\qquad \mathbf{N}=99\end{array}$ & $\begin{array}{c}\begin{array}{c}\text { Remote or very } \\
\text { remote }\end{array} \\
\quad \mathrm{N}=59\end{array}$ & $\begin{array}{c}\text { p- } \\
\text { value }^{* *}\end{array}$ \\
\hline Male & $130(82.3 \%)$ & $82(82.8 \%)$ & $48(81.4 \%)$ & $\mathrm{P}=0.832$ \\
\hline Mean age (SD)***; range [years] & 60.9 (12.4); 26 to 89 & 61.1 (12.7); 26 to 89 & 60.7 (12.0); 31 to 89 & $\mathrm{P}=0.852$ \\
\hline Caucasian & $134(84.8 \%)$ & 89 (89.9\%) & 45 (76.3\%) & $\mathrm{P}=0.037$ \\
\hline Born in Australia & $131(83.4 \%)$ & $80(80.8 \%)$ & $51(87.9 \%)$ & $\mathrm{P}=0.275$ \\
\hline Secondary or higher school education; $n=121$ & $90(74.4 \%)$ & $63(79.7 \%)$ & $27(64.3 \%)$ & $\mathrm{P}=0.140$ \\
\hline Employed & $77(48.7 \%)$ & $46(46.5 \%)$ & 31 (52.5\%) & $\mathrm{P}=0.288$ \\
\hline Living alone & $52(32.9 \%)$ & $32(32.3 \%)$ & $20(33.9 \%)$ & $\mathrm{P}=0.207$ \\
\hline With dependants & $24(15.2 \%)$ & $16(16.2 \%)$ & $8(13.6 \%)$ & $\mathrm{P}=0.819$ \\
\hline Private health insurance & $54(34.2 \%)$ & $29(29.3 \%)$ & $25(42.4 \%)$ & $\mathrm{P}=0.260$ \\
\hline ome protection cover & $2(1.3 \%)$ & $2(2.0 \%)$ & $0(0 \%)$ & $\mathrm{P}=0.529$ \\
\hline Life insurance & $19(12.0 \%)$ & $14(14.1 \%)$ & $5(8.5 \%)$ & $\mathrm{P}=0.325$ \\
\hline Income less then $\$ 20,000 ; n=107$ & 42 (39.3\%) & $23(35.4 \%)$ & $19(45.2 \%)$ & $\mathrm{P}=0.320$ \\
\hline Incidental finding & $11(7.0 \%)$ & $6(6.1 \%)$ & $5(8.5 \%)$ & $\mathrm{P}=0.748$ \\
\hline Squamous cell carcinoma & $156(100 \%)$ & 98 (100\%) & $58(100 \%)$ & l \\
\hline [NM Stage & & & & $\mathrm{P}=0.304$ \\
\hline & 17 (10.8\%) & $9(9.1 \%)$ & $8(13.8 \%)$ & \\
\hline HI & $24(15.3 \%)$ & $19(19.2 \%)$ & $5(8.6 \%)$ & \\
\hline III & $33(21.0 \%)$ & $20(20.2 \%)$ & $13(22.4 \%)$ & \\
\hline IV & $83(52.9 \%)$ & $51(51.5 \%)$ & $32(55.2 \%)$ & \\
\hline Treatment palliative & 40 (25.3\%) & 27 (27.3\%) & 13 (22.0\%) & $\mathrm{P}=0.571$ \\
\hline
\end{tabular}

This article is protected by copyright. All rights reserved. 


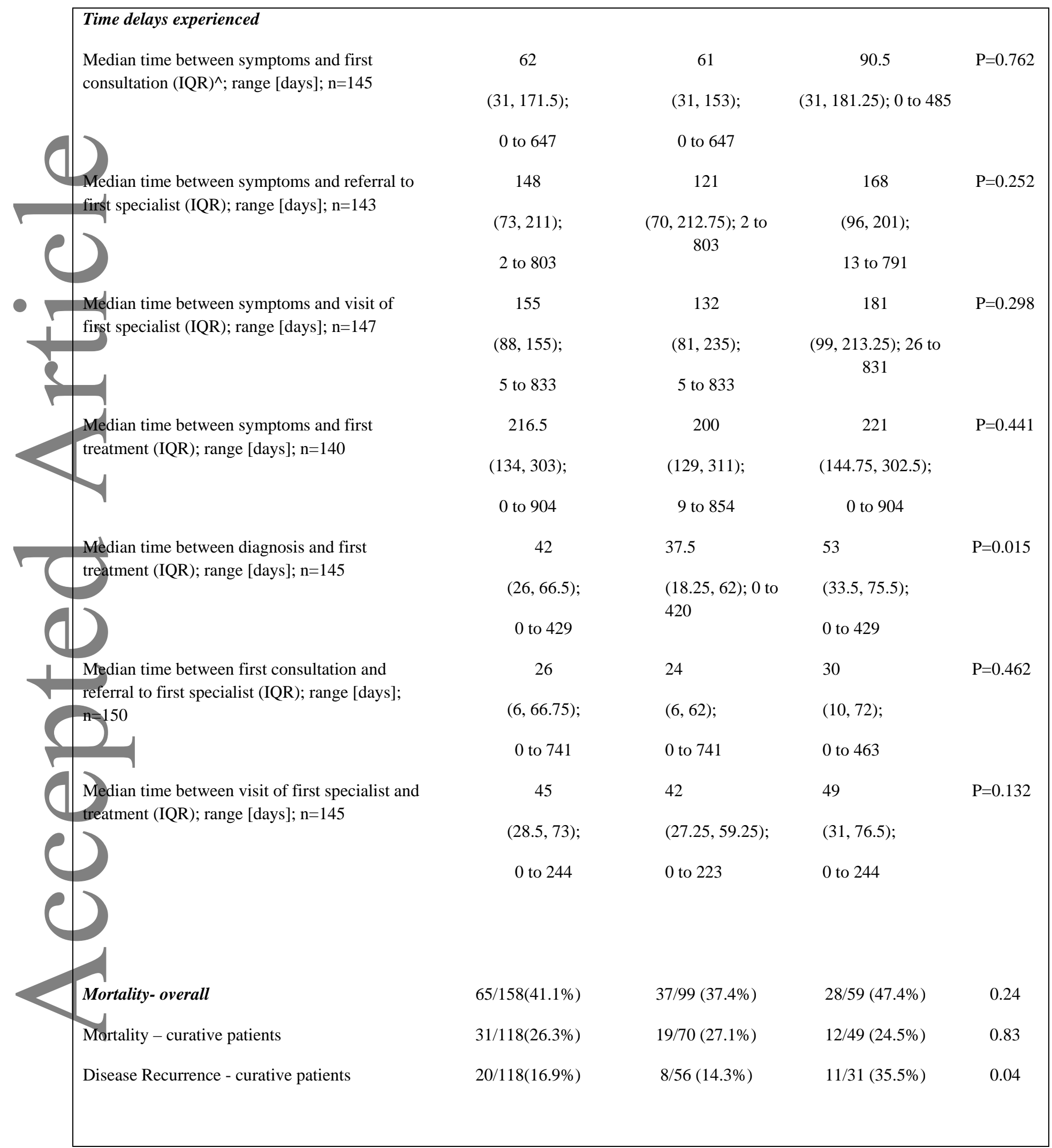

*Includes one person from a major city; ${ }^{* *}$ p-values are results of Fisher's exact tests, unpaired t-tests, and Mann-Whitney tests; ${ }^{* * *} \mathrm{SD}=$ standard deviation; ${ }^{\wedge} \mathrm{IQR}=$ inter-quartile range.

This article is protected by copyright. All rights reserved. 
$\wedge \wedge$ Chi squared

Table 2. Indigenous patients

Time delays experienced

Median time between symptoms and

first consultation (IQR)^; range [days]; $\mathrm{n}=145$

1

Median time between symptoms and referral to first specialist (IQR); range [ddys]; $\mathrm{n}=143$

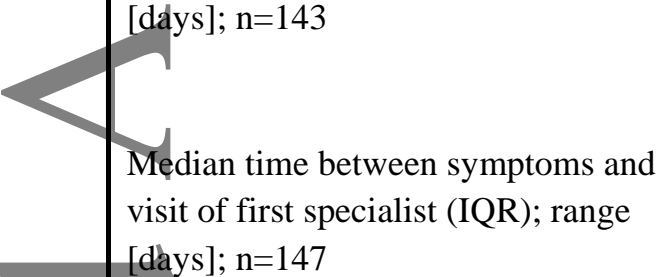

$\sim$

Median time between symptoms and first treatment (IQR); range [days]; $n=140$

Mediah time between diagnosis and first treatment (IQR); range [days];

$n=145$

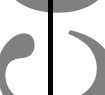

Median time between first consultation

and referral to first specialist (IQR);

ange [days]; $n=150$

Median time between visit of first

specialist and treatment (IQR); range

[days]; $\mathrm{n}=145$

\begin{tabular}{cccc}
\multicolumn{1}{c}{ Indigenous } & & & \\
No & Yes & p-value & p-value* \\
$(\mathbf{n}=\mathbf{1 3 5})$ & $(\mathbf{n}=\mathbf{2 3})$ & & \\
61 & 92 & $\mathrm{P}=0.218$ & $\mathrm{P}=0.236$ \\
$(31,155.25) ;$ & $(47.5,181) ;$ & & \\
0 to 485 & 13 to 647 & & \\
132 & 175 & $\mathrm{P}=0.472$ & $\mathrm{P}=0.514$ \\
$(73,212) ;$ & $(80,209) ;$ & & \\
2 to 791 & 13 to 803 & & \\
147.5 & 181 & $\mathrm{P}=0.501$ & $\mathrm{P}=0.546$
\end{tabular}

(87.75, 222.5); $\quad(88,240)$;

5 to $831 \quad 26$ to 833

$207.5 \quad 246.5 \quad \mathrm{P}=0.129 \quad \mathrm{P}=0.173$

(129, 293.25); $\quad$ (167.25, 327);

0 to $904 \quad 96$ to 854

$39 \quad 69.5 \quad \mathrm{P}=0.013 \quad \mathrm{P}=0.027$

(22, 62); $\quad(35.75,109)$;

0 to $429 \quad 0$ to 420

$26 \quad 19 \quad \mathrm{P}=0.329 \quad \mathrm{P}=0.260$

$(7,72) ; \quad(0,45.25)$;

0 to $741 \quad 0$ to 463

$42 \quad 57 \quad \mathrm{P}=0.031 \quad \mathrm{P}=0.030$

$(27,66.5) ; \quad(44.5,93.5)$;

0 to $244 \quad 1$ to 122

This article is protected by copyright. All rights reserved. 


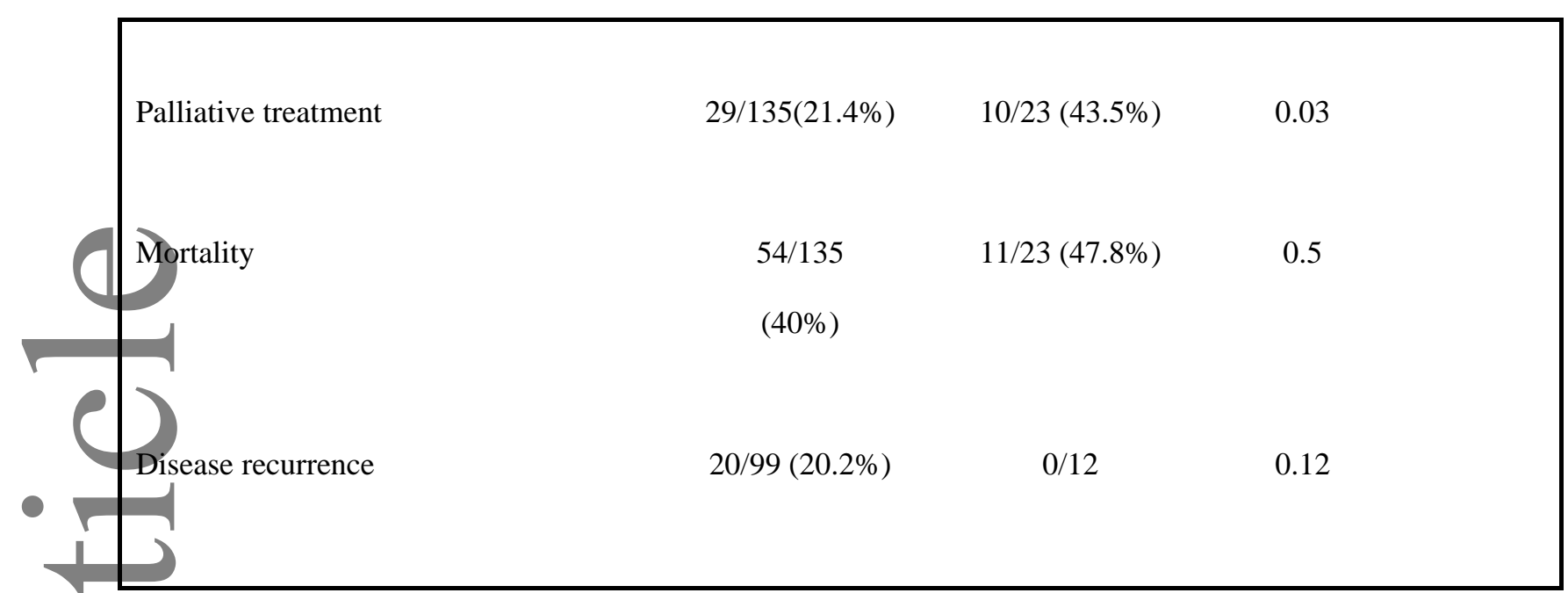
$\wedge$ IQR = inter-quartile range; *First p-value is result of Mann-Whitney test; ${ }^{* *}$ Second p-value
excluded 11 patients with incidental findings.

This article is protected by copyright. All rights reserved. 
Time between first consultation and referral to first specialist***
Location of residence

Townsville

Cairns or Mackay

All other
Reference

$$
0.461
$$

$-0.260,1.182$

$\mathrm{P}=0.209$

0.095, 1.413

0.754

Time between visit of first specialist and treatment $\wedge$

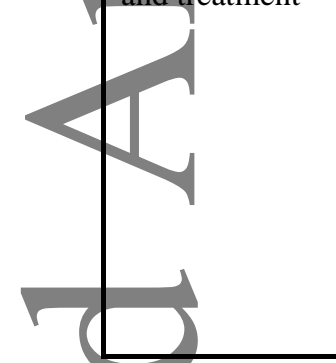

Location of residence

Townsville

Cairns or Mackay

All other
Reference

$-0.069,0.763$

$\mathrm{P}=0.102$

0.747

$0.265,1.229$

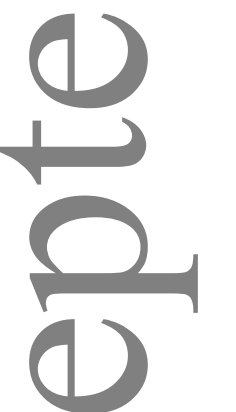

*Model based on 81 patients (missing: level of education $n=37$; income $n=51$; time delay $n=18$ ) ; model was adjusted for the confounding effects of ethnicity, employment status and whether a synchronous cancer was present.

**Model based on 116 patients (missing: level of education $n=37$; time delay $n=13$ ) ; model was adjusted for the confounding effects of ethnicity, and level of education.

***Model based on 150 patients (missing: time delay $n=8$ ) ; model was adjusted for the confounding effects of ethnicity and remoteness.

^Model based on 145 patients (missing: time delay $n=13$ ); model was adjusted for the confounding effects of remoteness.

This article is protected by copyright. All rights reserved. 